\title{
Battery Capacity Measurement and Analysis using Lithium Coin Cell Battery
}

\author{
Sung Park, Andreas Savvides, Mani B. Srivastava \\ Networked and Embedded Systems Laboratory \\ Electrical Engineering Departments, University of California, Los Angeles \\ \{spark, asavvide, mbs\}@ee.ucla.edu
}

\begin{abstract}
In this paper, we look at different battery capacity models that have been introduced in the literatures. These models describe the battery capacity utilization based on how the battery is discharged by the circuits that consume power. In an attempt to validate these models, we characterize a commercially available lithium coin cell battery through careful measurements of the current and the voltage output of the battery under different load profile applied by a micro sensor node. In the result, we show how the capacity of the battery is affected by the different load profile and provide analysis on whether the conventional battery models are applicable in the real world. One of the most significant finding of our work will show that DC/DC converter plays a significant role in determining the battery capacity, and that the true capacity of the battery may only be found by careful measurements.
\end{abstract}

\section{Keywords}

Embedded System, Battery, Power Estimation, Energy Estimation, DC/DC Converter, Coin Cell, Data Acquisition

\section{INTRODUCTION}

Proliferation of battery-powered mobile devices such as handhelds, cell phones, and pagers has given motivation to look for ways to prolong the lifetime of the battery. Furthermore, the slow improvement of the battery technology relative to the growth of power demand from these mobile devices has been fueling many studies in characterization and optimization of power usage of mobile devices so that the battery can be efficiently utilized. One of the vital pieces necessary in characterizing the power usage of the mobile system is the accurate battery model. An accurate battery model can reveal the efficiency of wireless protocols and power management schemes used in the mobile devices while an inaccurate model may tell a story far different from reality. However, this vital piece of the puzzle have remained a stumbling block for many of the electrical engineers and computer scientists due to batteries' complex technologies which involve many intricate highly non-linear electrochemical phenomenon. Moreover, many factors such as battery dimension, makeup of anode or cathode, and transport or diffusion rate of

Permission to make digital or hard copies of all or part of this work for personal or classroom use is granted without fee provided that copies are not made or distributed for profit or commercial advantage and that copies bear this notice and the full citation on the first page. To copy otherwise, or republish, to post on servers or to redistribute to lists, requires prior specific permission and/or a fee.

ISLPED '01, August 6-7, 2001, Huntington Beach, California, USA. Copyright 2001 ACM 1-58113-371-5/01/0008...\$5.00. active materials, that contribute to the characteristics of the battery have kept the battery technology in the domain of electrochemists.

In spite of these difficulties, there have been recent efforts $[1],[6],[7]$ to generalize these complexities of the battery by modeling batteries' inherent characteristics. These models range from simple linear model to a complex model that attempts to incorporate the "relaxation" phenomenon. So far, these models, though novel in conceptual sense, lacked the validation in the real world. Moreover, studying these models does not provide the bottom line for a mobile device designer who simply wants to find out what the maximum lifetime of his or her circuit will be. Much of this is contributed by the difficulties mentioned in previous paragraph. In an attempt to overcome these difficulties, in this paper we propose a technique that can be used in characterizing the battery capacity. The technique is to carefully measure the battery's current and voltage output for the duration of the battery lifetime as the embedded board consumes power from the battery. This in turn will provide accurate measurement of the battery capacity under different load profile generated by the embedded board. Not only the technique can help estimating the battery capacity, but it can also be used to validate some of the battery models that have been proposed to see whether the outcomes foreseen by those models are accurate in reality. One primary focus of the paper is to look at the impact of the DC/DC converter by finding out whether the capacity delivered to the circuit is actually same as what was consumed from the battery. The paper is organized as follows. We provide an overview of some of the battery models in interest in section 2 , and discuss how the measurement is done in section 3 . We include the results and the analysis of the measurement in section 4 , and we conclude the paper with section 5 .

\section{BATTERY MODELS}

Recent efforts in modeling the battery capacity are captured in this section. These models can also be viewed as different generations of battery models with later generations incorporating additional characteristics of the battery technology. The metrics that are used to indicate the maximum capacity of the battery is in the unit of Ah (Ampere*Hour). The metric is a common method used by the battery manufacturers to specify the theoretic total capacity of the battery. Knowing the current discharge of the battery and the

\footnotetext{
${ }^{\dagger}$ This paper is based in part on research performed under DARPA Power Aware Computing and Communications program through AFRL contract \# F30602-00-C-0154. The views and conclusions contained herein are those of the authors and should not be interpreted as necessarily representing the official policies or endorsements, either expressed or implied, of the DARPA, Air Force Rome Laboratory or the U.S. Government.
} 
total capacity in Ah, one can compute the theoretical lifetime of the battery using the equation , $T=\frac{C}{I}$, where $T=$ battery lifetime, $C=$ rated maximum battery capacity in $\mathrm{Ah}$, and $I=$ discharge current. More on this metric can be found in [5],[7].

The following subsections are brief descriptions of three battery capacity models that we consider.

\subsection{Linear Model $-1^{\text {st }}$ Generation}

In Linear Model, the battery is treated as linear storage of current. The maximum capacity of the battery is achieved regardless of what the discharge rate is. The simple battery model allows user to see the efficiency of the user's application by providing how much capacity is consumed by the user. The remaining capacity after operation duration of time $t_{d}$ can be expressed by the following equation.

Remaining capacity (in Ah) $=C=C^{\prime}-\int_{t=t_{0}}^{t_{0}+t_{d}} I(t) d t$, Eq.(1)

where $C^{\prime}$ is the previous capacity and $I(t)$ is the instantaneous current consumed by the circuit at time $t$. The Linear Model assumes that $I(t)$ will stay the same for the duration $t_{d}$, if the operation mode of the circuit does not change ( i.e. radio switching from receiving to transmit, CPU switching from active to idle, etc.. ) for the duration $t_{d}$. With these assumptions equation 1 simply becomes as the following.

$$
C=C^{\prime}-\int_{t=t_{0}}^{t_{0}+t_{d}} I(t) d t=C^{\prime}-\left.I \cdot t\right|_{t_{0}} ^{t_{0}+t_{d}}=C^{\prime}-I \cdot t_{d}, \quad \mathrm{Eq}(2)
$$

The total remaining capacity is computed whenever the discharge rate of the circuit changes. Being the most simplistic model, Linear Model falls short of portraying the behavior of a real life battery with characteristics such as rate dependent capacity and relaxation.

\subsection{Discharge Rate Dependent Model $-2^{\text {nd }}$ Generation}

While Linear Model assumes that the maximum capacity of the battery is unaffected by the discharge rate, Discharge Rate Dependent Model considers the effect of battery discharge rate on the maximum battery capacity. In [1] [5] [15], it is shown that battery's capacity is reduced as the discharge rate increases. In order to consider the effect of discharge rate dependency, we introduce factor $k$ which is the battery capacity efficiency factor that is determined by the discharge rate. The definition of $k$ is, $k=\frac{C_{e f f}}{C_{\max }}$, where $C_{e f f}$ is the effective battery capacity and $C_{\max }$ is the maximum capacity of the battery with both terms expressed in unit of Ah. In Discharge Rate Dependent Model, the equation 1 is then transformed to the following.

$$
C=k \cdot C^{\prime}-I \cdot t_{d}, \quad \text { Eq.(3) }
$$

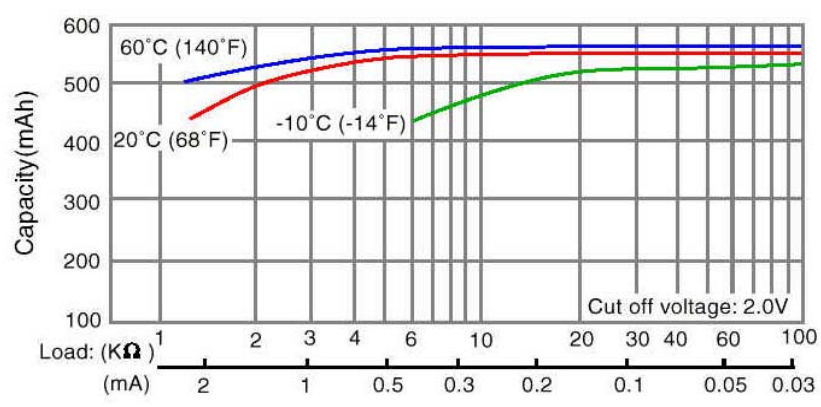

Figure 1. Capacity vs. Discharge Rate Curve for CR2354

The efficiency factor $k$ varies with the current $I$ and is close to one when discharge rate is low, but approaches 0 when the discharge rate becomes high. One way to find out what the $k$ value is for different current value $I$ is to use the table driven method introduced in [7]. With a table driven method, the factor $k$ can be looked up from a plot similar to figure 1 which can be obtained from battery manufacturer's data sheet [9]. The figure 1 plots the battery capacity $\left(C_{e f f}\right)$ versus different discharge rate $I$. Using the plot, whenever the remaining capacity is computed (equation 3 ), the factor $k$ can be obtained from the plot by looking at the total efficiency of the battery capacity for given current $I$. One shortfall of Discharge Rate Dependent Model is the fact that it does not portray the behavior of real battery by neglecting the effect of relaxation.

\subsection{Relaxation Model $-3^{\text {rd }}$ Generation}

Real-life batteries exhibit a general phenomenon called "relaxation" explained in [1],[5],[6]. When the battery is discharged at high rate, the diffusion rate of the active ingredients through the electrolyte and electrode falls behind. If the high discharge rate is sustained, the battery reaches its end of life even though there are active materials still available. However, if the discharge current from the battery is cutoff or reduced during the discharge, the diffusion and transport rate of active materials catches up with the depletion of the materials. This phenomenon is called relaxation effect, and it gives the battery chance to recover the capacity lost at high discharge rate. For a realistic battery simulation, it's important to look at the effects of relaxation as it has effect of lengthening the lifetime of the battery. [1] introduces an analytical model which takes discharge rate as input and computes the battery voltage over the simulation duration. On the other hand, [6], [8] introduce a stochastic model, where the recovery is modeled as a change of state which occurs at a pre-set discharge rate. Obtained using the analytical model from [4], the curve in figure 2 demonstrates the effect of

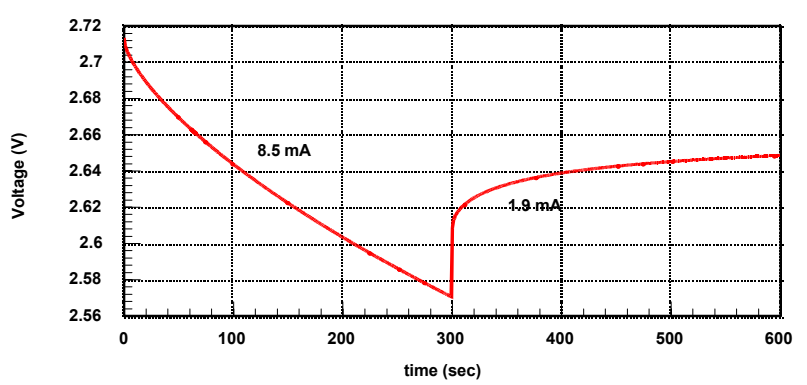

Figure 2. Recovery Effect of Relaxation Model 
relaxation. In figure 2 the battery voltage recovers when the battery discharge rate is reduced to $1.9 \mathrm{~mA}$ after being discharged at $8.5 \mathrm{~mA}$. Although the relaxation model is the most comprehensive model that closely describes the behavior a real battery, there exists considerable difficulty in implementing such model since the relaxation effect involves many electrochemical and physical properties of the battery. This is demonstrated by the analytical model from [4] which contains over 50 electrochemical and physical parameters of inputs that need to be measured separately for different types of battery.

This difficulty gives motivation for estimating the battery capacity based on a measurement based approach.

\section{MEASUREMENT SETUP}

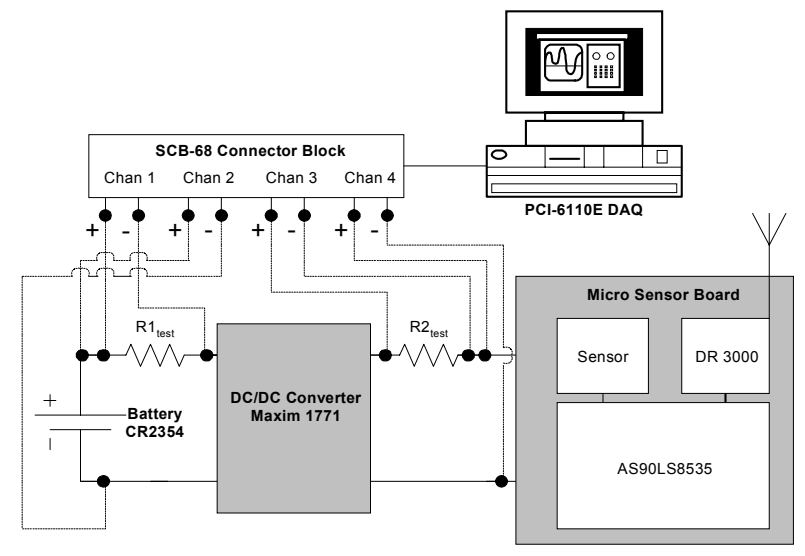

Figure 3. Battery Measurement Setup

In this paper, we propose an entirely different approach of estimating the battery capacity. The approach is to measure the current and voltage output of the battery as an embedded board consumes current out from the battery. The measurement is performed for different load profile until the battery's cutoff voltage is reached and the effective capacity of battery is computed.

Figure 3 shows the setup of the battery capacity measurement performed on a lithium coin cell, CR2354. There are total of 4 channels that monitor the current and voltage output of the battery (Chan1 and Chan2), and the current and the voltage output (Chan3 and Chan4) of DC/DC Converter. In order to measure the current, we measure the minute voltage drop across two small resistors, $\mathrm{R} 1_{\text {test }}$ and $\mathrm{R} 2_{\text {test }}$, which are $2.2 \Omega$ high precision resistors. While Chan 1 and 2 monitor how much current and power are consumed from the battery, Chan 3 and 4 measures how much current and energy are actually delivered to the sensor board based on different load profiles. The values from the four channels are recorded to PC that encloses the PCI-6110E DAQ board. The measurement starts as a freshly charged (factory sealed) battery is placed on the circuit and micro sensor board is turned on. The measurement is stopped when the battery reaches the minimum voltage of $2.0 \mathrm{~V}$ at which point $\mathrm{DC} / \mathrm{DC}$ converter no longer supplies required voltage to the micro sensor board. The followings are the description of each components used in the measurement.

\subsection{Micro Sensor Board}

Table 1. Node States And Current Consumption at 3.3V

\begin{tabular}{|c|c|c|c|}
\hline Operation Mode & AVR State & RFM State & Avg Current \\
\hline Tx & $O N$ & $T X$ & $12.2 \mathrm{~mA}$ \\
\hline $\mathrm{Rx}$ & $O N$ & $R X$ & $9.0 \mathrm{~mA}$ \\
\hline Idle & $O N$ & $S L E E P$ & $7.8 \mathrm{~mA}$ \\
\hline Sleep & $S L E E P$ & $S L E E P$ & $4.2 \mathrm{~mA}$ \\
\hline
\end{tabular}

The wireless sensor node we have built uses an RFM DR3000 radio module [3] and an AVR 90LS8535 microcontroller [2] from Atmel. The radio can transmit at 2 different data rates $(2.4 \mathrm{Kbps}$ and $19.2 \mathrm{Kbps}$ ) and supports OOK and ASK modulation. The microcontroller has 8 Kbytes of flash memory, 512 bytes of SRAM and 512 bytes of EEPROM. It is also equipped with an 8 channel 10 ADC, a programmable UART and an SPI bus. Our current implementation has a prototyping area that can support a wide variety of add on sensors such as magnetometers, thermometers, accelerometers and light sensors. This node is a variant of the widely used Smart Dust [14] nodes from UC Berkeley. The board also includes an array of ultrasound sensors that can be used to infer node location. Our main focus is the different power modes of the node that are shown in table 1.

\subsection{Lithium Coin Cell Battery - CR2354 ${ }^{1}$}

For the measurement, CR2354 lithium coin cell is used. With high energy density and relatively flat discharge characteristics, lithium batteries are widely used in mobile devices such as cellular phones, digital cameras, and PDAs. Especially for micro sensor nodes, small form factor is an essential necessity and the size of lithium coin cell batteries satisfies the stringent requirement of micro sensor nodes. Table 2 provides the manufacturer's specification of CR2354 which can be obtained from [9].

Table 2. CR2354 Specification

\begin{tabular}{|c|c|c|c|c|}
\hline $\begin{array}{c}\text { Output } \\
\text { Voltage }\end{array}$ & $\begin{array}{c}\text { Cutoff } \\
\text { Voltage }\end{array}$ & $\begin{array}{c}\text { Max. } \\
\text { Capacity }\end{array}$ & $\begin{array}{c}\text { Dimension } \\
\text { Diam.xHt. }\end{array}$ & Wt \\
\hline \hline $3.0 \mathrm{~V}$ & $2.0 \mathrm{~V}$ & $560 \mathrm{mAh}$ & $23.0 \mathrm{~mm} \times 5.4 \mathrm{~mm}$ & $5.9 \mathrm{~g}$ \\
\hline
\end{tabular}

\subsection{DC/DC Converter (MAXIM 1771²)}

As the battery is discharged, the voltage across the battery constantly decreases. When the battery is directly connected to a VLSI circuit without any form of voltage regulator, the circuits' performance will start to degrade as the voltage across the battery decreases. Moreover, when the battery's voltage reaches the minimum input voltage required by the circuit, the circuit will stop functioning even though there may be some capacity left in the battery. It is the role of $\mathrm{DC} / \mathrm{DC}$ converter to provide a constant voltage to the circuit while utilizing complete capacity of the battery. One type of DC/DC converter is called "switching regulator". A switching regulator is a circuit that uses an inductor, a transformer, or a capacitor as an energy-storage element to transfer energy from input to output in discrete packets [13]. Switching regulators can be configured to be either step up (boost) or step down (buck) or inverting with respect to the input voltage [12]. Due to their efficiency and versatility, switching regulators have been popular in battery powered mobile devices. When considering battery capacity measurement, it is important to look at the operation DC/DC

\footnotetext{
${ }^{1}$ Manufactured by Panasonic

${ }^{2}$ Manufactured by Maxim Integrated Products, Inc.
} 
converters since the converters discharge the battery based on its own voltage regulator function. By doing so, DC/DC converters completely change the load profile generated by the VLSI circuit. In our measurement, we look at the both input and the output of the DC/DC converter to see how the operation DC/DC converter impacts the overall performance of the battery.

The DC/DC converter used in the measurement is MAXIM 1771 step up switching controller, which can provide a constant $3.3 \mathrm{~V}$ from the minimum input of 2V. Maxim 1771 uses the currentlimited pulse-frequency-modulation (PFM) where the charge cycle is cut-off when a predetermined peak inductor current is reached and it remains at the cut-off stage for a pre-determined fixed duration (called one-shot time constant)[13]. The effect of DC/DC Converter on the battery current and voltage output will be shown in section 4 .

\subsection{PCI-6110E 'DAQ Board with SCB-68 ${ }^{3}$ Connection Block}

PCI-6110E board is a real time data acquisition board that can scan up to 4 input channels simultaneously. The board is equipped with 12 bit ADC per channel with sampling rate of up to $5 \mathrm{Meg}$ Samples/sec. The board uses SCB-68 connection block to connect to the actual input channel. For the most of the measurement the board was configured to scan at $5 \mathrm{k}$ samples/sec for each channel and some of the detailed measurements were done at $25 \mathrm{k}$ samples/second. Also, in order to measure the minute voltage drop across $\mathrm{R} 1_{\text {test }}$ and $\mathrm{R} 2_{\text {test }}$, the precision of the board was configured to 97.66 $\mu \mathrm{V}$ for Chan1 and Chan3. The precision of Chan 2 and 4 was set at $2.44 \mathrm{mV}$. More information on the DAQ board can be found from [10], [11].

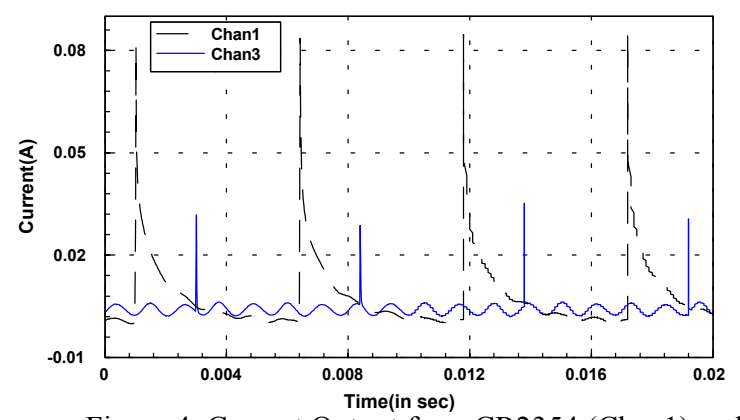

Figure 4. Current Output from CR2354 (Chan1) and DC/DC Converter (Chan3) during Sleep Mode - Snap Shot

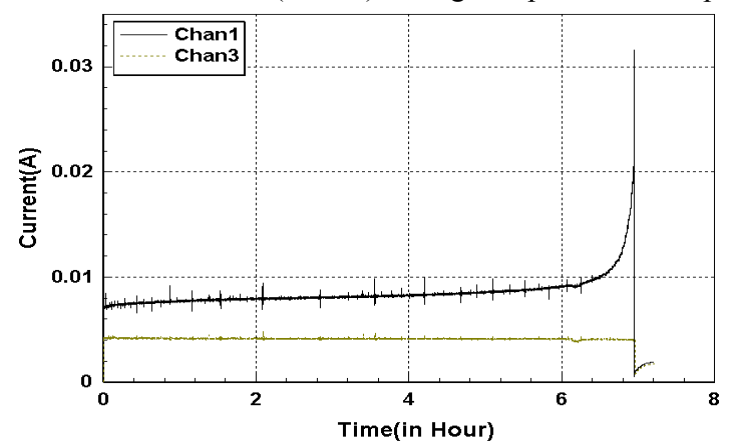

Figure 6. Average Current Output from CR2354 (Chan1) and DC/DC Converter (Chan3) during Sleep Mode - Complete

\section{RESULTS}

This section shows the performance of the battery by configuring the sensor board into different operation modes displayed in table 1. We look at how the battery capacity is affected by the discharge rate, DC/DC converter's voltage regulation function, and discharge profile of the sensor board. Figure 4 and 5 show snapshots $(20 \mathrm{msec})$ of the current and voltage output observed from all 4 channels. One noticeable effect shown in figure 4 is that the current drawn by the sensor board (Chan3) is quite different from the current from the battery (Chan1) as the current drawn by the sensor stays around constant $4 \mathrm{~mA}$ while the current drawn out of the battery ranges from $80 \mathrm{~mA}$ to $0 \mathrm{~mA}$. The current discharge pulse seen at Chan 1 is the direct result of the DC/DC converters' PFM switching function which completely changes the load profile of the sensor board. This difference can also be seen from the voltage outputs shown in figure 5. The relaxation effect plays a role in the battery as the voltage across the battery (Chan2) drops when the charge cycle starts then recovers when the discharge current is cutoff. Compared to the voltage seen at Chan2, the voltage output of the DC/DC converter (Chan4) doesn't vary much as it is kept above $3.3 \mathrm{~V}$.

These differences between the output seen from the battery and the output seen by the sensor board becomes clearer when the current and voltage values are observed for the complete cycle of the battery life time. Figure 6 and 7 show how the battery is utilized as the sensor board stays at sleep mode. The curves in figure 6 and 7 are smoothed by averaging the samples collected during one second interval. In figure 6 the current output from the battery increases as much as $32 \mathrm{~mA}$ when the DC/DC

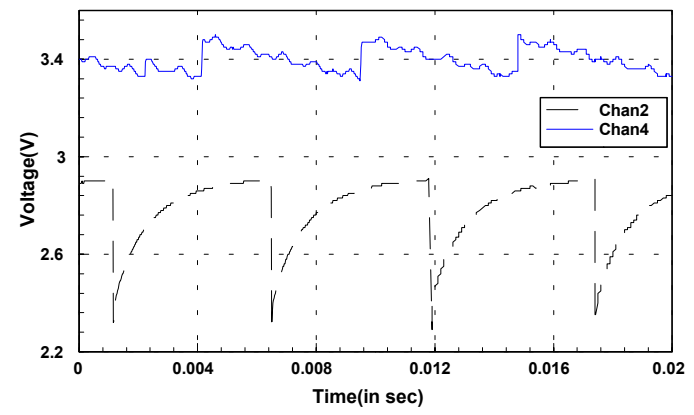

Figure 5. Voltage Output from CR2354 (Chan2) and DC/DC Converter (Chan4) during Sleep Mode - Snap Shot

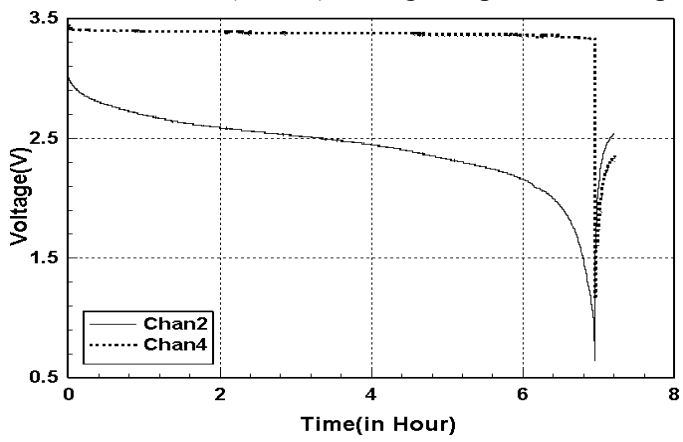

Figure 7. Average Voltage Output from CR2354 (Chan2) and DC/DC Converter (Chan4) during Sleep Mode - Complete

\footnotetext{
${ }^{3}$ Product of National Instruments Corp.
} 
Table 3. Battery Utilization for Various Sensor Operation Modes

\begin{tabular}{|c|c|c|c|c|c|c|}
\hline $\begin{array}{l}\text { Operating } \\
\text { Mode }\end{array}$ & $\begin{array}{c}\text { Total } \\
\text { Lifetime }\end{array}$ & $\begin{array}{c}\text { Capacity consumed } \\
\text { from Battery }\end{array}$ & $\begin{array}{c}\text { Capacity delivered } \\
\text { to Sensor }\end{array}$ & $\begin{array}{c}\text { Energy consum ed } \\
\text { from Battery }\end{array}$ & $\begin{array}{c}\text { Capacity delivered } \\
\text { to Sensor }\end{array}$ & $\begin{array}{c}\text { DC / DC converter } \\
\text { efficiency }\end{array}$ \\
\hline 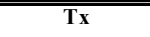 & $=.33 \mathrm{hr}$ & $8.1 \mathrm{mAh}$ & $4.1 \mathrm{~m} \mathrm{Ah}$ & $70.0 \mathrm{~J}$ & $449.1 \mathrm{~J}$ & 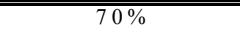 \\
\hline $\mathbf{R x}$ & $.88 \mathrm{hr}$ & $15.8 \mathrm{mAh}$ & $7.9 \mathrm{~m} \mathrm{Ah}$ & $135.2 \mathrm{~J}$ & $96.1 \mathrm{~J}$ & $71 \%$ \\
\hline Id le & $1.2 \mathrm{~h} \mathrm{r}$ & $18.0 \mathrm{~m} \mathrm{Ah}$ & $9.0 \mathrm{~m} \mathrm{~A} \mathrm{~h}$ & $154.0 \mathrm{~J}$ & $109.0 \mathrm{~J}$ & $71 \%$ \\
\hline S le ep & $7.0 \mathrm{hrs}$ & $59.1 \mathrm{~m} \mathrm{Ah}$ & $28.8 \mathrm{~m} \mathrm{Ah}$ & $505.1 \mathrm{~J}$ & $350.7 \mathrm{~J}$ & $69 \%$ \\
\hline
\end{tabular}

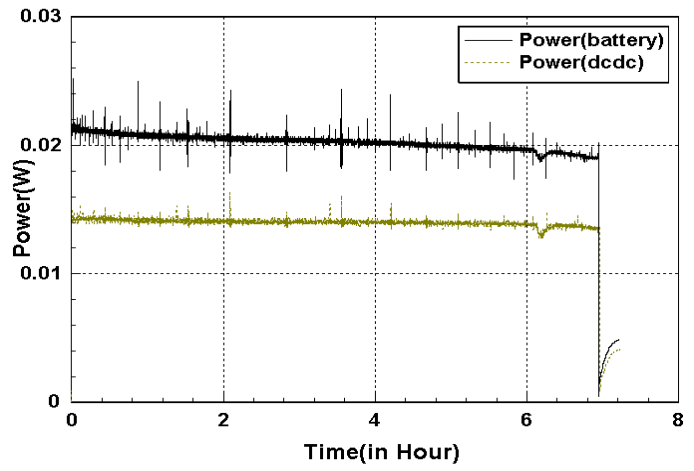

Figure 8. Power Output from CR2354 and DC/DC Converter (Chan3) during Sleep Mode

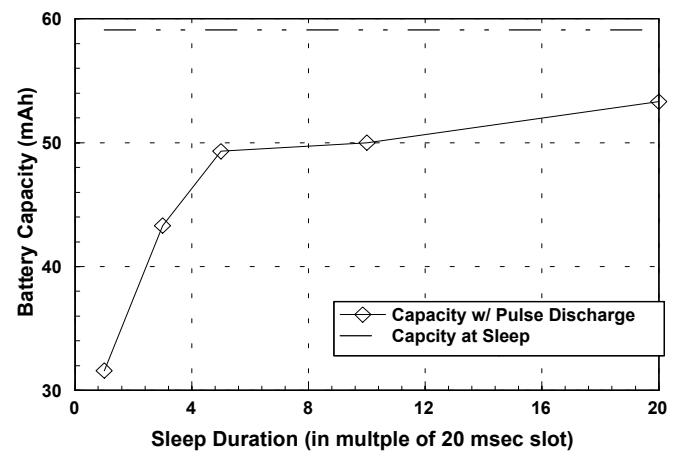

Figure 10. Impact of Pulse Discharging on Battery

converter pulls more and more current out from the battery while the voltage across the battery drops (figure 7). This increase in current discharge continues until it reaches the cutoff points where DC/DC converter shuts down which occurs after approximately 7 hours. One interesting thing to notice in figure 6 is that the total capacity of $28.8 \mathrm{mAh}$ (area under the curve) delivered to the sensor board is much smaller than what the $\mathrm{DC} / \mathrm{DC}$ converter consumed from the battery which is $59 \mathrm{mAh}$. This difference can be explained by studying the power curves displayed in figure 8 . Figure 8 plots the power consumed from the battery and the power delivered to the sensor board which can be computed by multiplying the current and the voltage plot. The decrease in the voltage across the battery (Chan2 in figure 7 ) is matched by the increase in the current drawn from the battery (Chan1 in figure6) to give relatively constant power consumption over the course of the battery lifetime. Another factor that contributes to the difference in the current capacity is the efficiency factor of the DC/DC converter. This is demonstrated by the difference in energy (area under the curve) between the two curves shown in figure 8 .

In table 3 the differences between what is consumed from the battery and what is actually delivered to the sensor board at

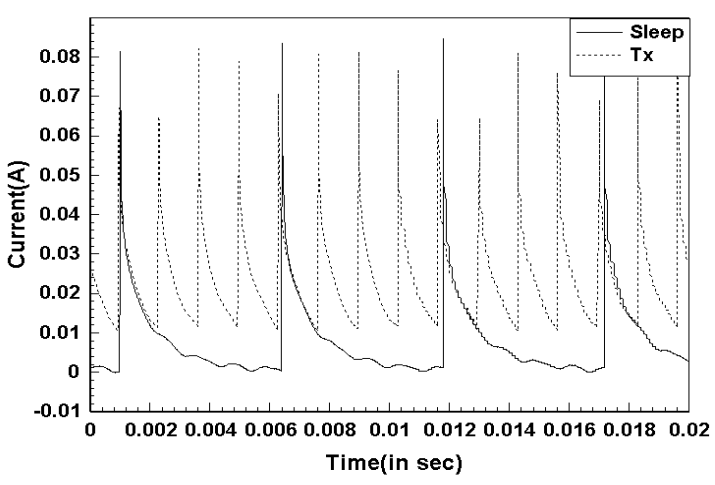

Figure 9. Current drawn from the battery during Sleep Mode and Tx Mode

different operating modes are listed. One trend that can be seen from the table is the characteristic that coincides with the discharge rate dependent model. Higher the discharge current, less capacity is utilized from the battery. This result comes despite the fact that the actual current discharge from the battery doesn't stay constant but fluctuates in discharge and relaxation cycles due to the PFM function implemented in DC/DC converter. Looking closely at the discharge curves in figure 9, it can be seen that when the sensor board draws a high current (in Tx mode), the battery doesn't have a chance to relax as the DC/DC converter pumps the current at high rate whereas in Sleep mode the rate of discharging cycle is much lower thus giving the battery more time to relax.

Shown in figure 10 is the effect of pulse discharging as the sensor board cycles between Rx mode and the Sleep mode. This case measures the battery capacity when a TDMA scheme is used with the sensor board which will be switching between the Rx mode and the Sleep mode within a fixed TDMA frame. For the measurement, the Rx duration is set at a fixed slot of $20 \mathrm{msec}$ and the Sleep duration was varied (in multiple of $20 \mathrm{msec}$ ) to observe what impacts the pulse discharging has on the battery capacity. The result shows that there is $100 \%$ increase in the battery capacity utilization when every Rx slot is followed by one Sleep slot (1:1 ratio). At 1:5 ratio, there is almost $250 \%$ improvement on the battery utilization. The increase in the battery utilization is sustained with longer duration of Sleep modes but quite never reaches the battery utilization achieved for Sleep modes even at 1:20 ratio. The plot shown in figure 10 can be used in estimating the effective battery capacity of a wireless device that uses a TDMA scheme with fixed schedules.

\section{CONCLUSION}

In this paper we have looked at various battery models that have been introduced so far. Though these models give us qualitative insights into how battery's capacity is influenced by multiple factors, they do not provide a simple way of finding out what the true battery capacity running in different operation modes. One approach that we have taken in this paper is based on a 
measurement approach, where we measure the capacity of the battery under different load profile to determine what the realized capacity of the battery is. The result shows that there are many factors such as the discharge rate, the discharge profile (constant vs. pulsing), and the DC/DC converter that govern the effective battery capacity. Especially, the role of the DC/DC converter in determining the battery capacity is a key factor that the conventional battery models don't account for. Since DC/DC converter changes the load profile generated by the sensor board based on its own voltage regulator function, we argue that the realistic battery model should consider the impact of DC/DC converter on the battery capacity. The result also suggests that a meaningful estimation of battery capacity can be achieved by measurements and not by just looking up what the manufacturer specifies. Moreover, when studying a low power design, just considering the energy number may not be sufficient as the battery lifetime has a profound dependency on how the battery is discharged. Our technique introduced in this paper provides a reasonable way of estimating the effective battery capacity and the means of justifying the low power design.

\section{REFERENCES}

[1] T. F. Fuller, M. Doyle, J. Newman, "Simulation and Optimization of the Dual Lithium Ion Insertion Cell," Journal of Electrochem. Soc., vol. 141, no. 4, Apr. 1994, pp. $1-10$.

[2] Atmel AS90LS8535 product website, http://www.atmel.com/atmel/products/prod200.htm last accessed on $2 / 8 / 2001$

[3] RFM Software Designer's Guide: http://www.rfm.com/corp/apnotes.htm last access on $2 / 8 / 2001$

[4] John Newman's Website: http://www.cchem.berkeley.edu/ jsngrp/ last accessed on $2 / 8 / 2001$.
[5] H.D. Linden, Handbook of Batteries, $2^{\text {nd }}$ ed., McGrawHill, New York 1995.

[6] C. F. Chiasserini and R. R. Rao, "Pulsed battery discharge in communication devices," Proceedings of Mobicom 99, Seattle, August 1999

[7] T. Simunic, L. Benini, G. De Micheli, "Energy-Efficient Design of Battery-Powered Embedded Systems," Proceedings of International Symposium on Low Power Electronics and Design, pp 212-217, Piscataway, August 1999

[8] D. Panigrahi, C. Chiasserini, S. Dey, R. Rao, A. Raghunathan, and K. Lahiri, "Battery Life Estimation of Mobile Embedded System", $14^{\text {th }}$ International Conference on VLSI Design, 2001

[9] Panasonic Lithium Coin Data Sheet: http://www.panasonic.com/industrial_oem/battery/battery_oe $\mathrm{m} / \mathrm{chem} /$ lith/lith.htm last accessed on $2 / 8 / 2001$.

[10] National Instruments Corp., PCI-6110E/6111E User's Manual, April 1998 ed., Austin TX 1998

[11] National Instruments Corp., NI-DAQ User Manual for PC Compatibles, January 2000 ed., Austin TX 2000

[12] M. D. Bruce, "Step-up/Step-down converters power small portable systems", EDN, February 31994

[13] Maxim Integrated Products, Inc., DC-DC Converter Tutorial, http://dbserv.maximic.com/tarticle/view_article.cfm?article_id=93 last access on $2 / 12 / 2001$

[14] M. Kahn, R.H. Katzand, K.S.J Pister, "Mobile Networking for Smart Dust", ACM|IEEE Int. Conf. on Mobile Computing and Networking, Seattle, WA, Aug 1999

[15] M. Pedram, Q. Wu, "Battery-Powered Digital CMOS Design", Proceedings of Design, Automation and Test in Europe Conference and Exhibition, Munich, Germany, March 1999 